\title{
Comparison of Network Criticality, Algebraic Connectivity, and Other Graph Metrics
}

\author{
Alireza Bigdeli \\ University of Toronto \\ 10 King's College Road \\ Toronto, Canada
}

\author{
Ali Tizghadam ${ }^{\dagger}$ \\ University of Toronto \\ 10 King's College Road \\ Toronto, Canada
}

\author{
Alberto Leon-Garcia ${ }^{\ddagger}$ \\ University of Toronto \\ 10 King's College Road \\ Toronto, Canada
}

\begin{abstract}
The study of robustness and connectivity properties are important in the analysis of complex networks. This paper reports on an effort to compare different network topologies according to their algebraic connectivity, network criticality, average node degree, and average node betweenness. We consider different network types and study the behavior of these various metrics as scale is increased. Based on extensive simulations, we suggest some guidelines for the design and simplification of networks. The main finding is that, algebraic connectivity, network criticality, average degree, and average node betweenness capture different properties of a graph. Depending on the nature of the problem at hand, one needs to select which one is appropriate to use as the main metric for network analysis.
\end{abstract}

\section{Keywords}

graph theory, graph spectrum, network robustness

\section{INTRODUCTION}

In order to develop guidelines for the simplification of complex networks, we need to study their connectivity and robustness properties. The connectivity of a graph can be quantified by algebraic connectivity [3], the second smallest eigenvalue of the Laplacian matrix of the graph. In order to quantify the robustness of a network, we need to precisely define the robustness. In this paper a network is said to be robust if its performance is not sensitive to the changes in topology, traffic or community of interest (the set of active sources/sinks for traffic). Based on this definition of robustness, we have proposed a metric, network criticality [10] to quantify the robustness of a network.

\footnotetext{
*Email: alireza.bigdeli@utoronto.ca

†Email: ali.tizghadam@utoronto.ca

†Email: alberto.leongarcia@utoronto.ca
}

Permission to make digital or hard copies of all or part of this work for personal or classroom use is granted without fee provided that copies are not made or distributed for profit or commercial advantage and that copies bear this notice and the full citation on the first page. To copy otherwise, to republish, to post on servers or to redistribute to lists, requires prior specific permission and/or a fee.

SIMPLEX'09, July 1, 2009, Venice, Italy.

Copyright 2009 ACM 978-1-60558-704-2/09/07 ...\$10.00.
In this paper we investigate and compare properties of algebraic connectivity and network criticality, along with node degree and node betweenness, and provide some guidelines for designing and simplifying complex networks based on desired connectivity and robustness properties.

A wealth of literature is available on network connectivity, robustness, and different related aspects. In [2] robustness of network topologies is studied. Graph-theoretic concepts are used to investigate which network topologies are the most robust. The authors argue that "node connectivity" is the most useful metric in graph theory to study the robustness problem. They examine the relationship between node connectivity and the degree of symmetry of a network and they suggest that it is important for robust networks to satisfy node similarity and optimal connectivity conditions. Two nodes are similar if there is an automorphism that can map one to the other. A network is node similar if all of its nodes are similar. A graph is optimally connected if its node connectivity is equal to the link connectivity metric and both are equal to the minimum node degree of the graph. [2] investigates the relationship between these conditions, and arrives at the result that a network provides maximum resistance to node destruction if it is both node-similar and optimally connected. The paper then describes a number of ways to design robust networks satisfying these conditions.

[1] introduces a new measure of symmetry, symmetry ratio of a network. This metric is defined to be the ratio of the number of distinct eigenvalues of the network to the diameter. This metric is used to study the robustness of a network topology in the face of targeted attacks.

In [9] we proposed a framework for robust routing in core networks based on the idea of "link criticality" and "path criticality". Further development of the idea of criticality is studied in [10], where a mathematical framework for the definition of network criticality is given within the context of Markov chain theory.

From a robustness point of view the algebraic connectivity is investigated in $[6,5]$. In $[6]$ the algebraic connectivity is studied in relation to graph's robustness to node and link failures. Three types of network topologies are considered: the random graph of Erdos-Renyi, the small-world graph of Watts-Strogatz and the scale-free graph of Barabasi-Albert, and through extensive simulations with the three complex network models, it is shown that the algebraic connectivity is not trivially related to the robustness to node and link failures. The authors have shown that in some cases the speed of increasing the algebraic connectivity is much lower than the speed of increasing node connectivity and that this 
is very dependent on the particular complex network model.

The goal of this paper is to investigate the behavior of network criticality when node connectivity increases and compare the results to those of $[6,5]$. We believe that this may help find networks that are both robust and simple while providing a desired level of connectivity.

The paper is structured as follows. Section 2 summarizes our previous results on network criticality. Section 3 provides the relationship between network criticality and algebraic connectivity. In section 4 a thorough set of simulations are conducted to compare the properties of network criticality and algebraic connectivity, and an analysis of the results is presented. In section 5 conclusions are provided.

\section{NETWORK CRITICALITY}

In this section we summarize the results of our previous work on robustness [10].

\subsection{Network Model}

We model a network with an undirected weighted graph $G=(N, E, W)$ where $\mathrm{N}$ is the set of nodes, $E$ is the set of graph links, and $W$ is the weight matrix of the graph. Throughout this paper we assume that $\mathrm{G}$ is a connected graph.

Consider a finite-state irreducible Markov Chain with transition probabilities $p_{i j}$ of transitioning from state $i$ at time $t$ to state $j$ at time $t+1$ (discrete time). The Markov chain can be represented by a state transition diagram with states as nodes in a graph and edges corresponding to allowable transitions, and labels associated with the edges denoting the transition probabilities. The Markov chain can also be viewed as a random walk on the n-node graph with nextstep transition probabilities $p_{i j}$ according to the following rule:

$$
p_{i j}=\left\{\begin{array}{c}
\frac{w_{i j}}{\sum_{k \in A(i)} w_{i k}} \text { if } j \in A(i) \\
0 \quad \text { otherwise }
\end{array}\right.
$$

where $A(i)$ is the set of adjacent nodes of $i$ and $w_{i k} \geq 0$ is the weight of link $(i, k)$.

We are interested in quantifying the betweenness of a node in the random-walk corresponding to a Markov chain. The original definition of random-walk betweenness is given in [7], but here we use a modified version defined in [10]. Consider the set of trajectories that begin at node $s$ and terminate when the walk first arrives at node $d$, that is, destination node $d$ is an absorbing node. We define the betweenness $b_{s k}(d)$ of node $k$ for the $s-d$ trajectories as the average number of times node $k$ is visited in trajectories from $s$ to $d$.

Let $B_{d}=\left[b_{s k}(d)\right]$ be the $n \times n$ matrix of betweenness metrics of node $k$ for walks that begin at node $s$ and end at node $d$. Further, let $P_{d}$ be the matrix of transition probabilities when the random walk is modified so that state $d$ is an absorbing state. We use $P(i \mid j)$ to show the truncated $(n-1) \times(n-1)$ matrix that results from removing $i^{\text {th }}$ row and $j^{\text {th }}$ column of matrix $P$. It is shown in [10] that:

$$
B_{d}(d \mid d)=\left(I-P_{d}(d \mid d)\right)^{-1}
$$

\subsection{Definition of Network Criticality}

We now introduce network criticality, the metric that we proposed in [10], to quantify the robustness of a network. We start by defining node/link criticality.
Node criticality is defined as the ratio of the random-walk betweenness of a node to its weight (weight of a node is defined as the sum of the weights of its incident links). Link criticality is similarly defined as the ratio of the betweenness of a link to its weight.

Let $\eta(k)$ be the criticality of node $k$ and $\eta_{i j}$ be the criticality of link $l=(i, j)$. It is shown in [10] that $\eta_{i}$ and $\eta_{i j}$ can be obtained by the following expressions:

$$
\begin{aligned}
\tau_{s d} & =l_{s s}^{+}+l_{d d}^{+}-2 l_{s d}^{+} \text {or } \tau_{s d}=u_{s d}^{t} L^{+} u_{s d} \\
\tau & =\sum_{s} \sum_{d} \tau_{s d}, \quad \hat{\tau}=\frac{1}{n(n-1)} \tau \\
\eta(k) & =\frac{b_{k}}{W_{k}}=\frac{1}{2} \tau=\frac{n(n-1)}{2} \hat{\tau} \\
\eta_{i j} & =\frac{b_{i j}}{w_{i j}}=\tau=n(n-1) \hat{\tau} \\
\frac{b_{s k}(d)}{W_{k}} & =l_{s k}^{+}-l_{s d}^{+}-l_{d k}^{+}+l_{d d}^{+}
\end{aligned}
$$

where $L^{+}=\left[l_{i j}^{+}\right]$is the Moore-Penrose inverse of graph Laplacian matrix $\mathrm{L}, \mathrm{n}$ is the number of nodes, and $u_{i j}=$ $\left[\begin{array}{lllllll}0 & \ldots & 1 & \ldots & -1 & \ldots & 0\end{array}\right]^{t}$ ( 1 and -1 are in $i^{\text {th }}$ and $j^{\text {th }}$ position).

OBSERVATION 2.1. Equations 3 to 6 show that node criticality $\left(\eta_{k}\right)$ and link criticality $\left(\eta_{i j}\right)$ are independent of the node/link position and only depend on $\tau$ (or $\hat{\tau}$ ) which is a global quantity of the network.

DEFINITION 2.2. We refer to $\tau$ as the network criticality and $\hat{\tau}$ as normalized network criticality. In this paper our experiments are based on normalized network criticality

One can see that $\hat{\tau}$ is a global quantity on network graph G. Equations 5 and 6 show that node (link) betweenness consists of a local parameter (weight) and a global metric (network criticality). $\hat{\tau}$ can capture the effect of topology and community of interest via betweenness, and the effect of traffic via weight (by appropriate definition of weight). The higher the betweenness of a node/link, the higher the risk of using the node/link. Furthermore, one can define node/link capacity as the weight of a node/link, then the higher the weight of a node/link, the lower the risk of using the node/link. Therefore network criticality can quantify the risk of using a node/link in a network which in turn indicates the degree of robustness of the network.

OBSERVATION 2.3. It turns out that the value of pair-wise criticality, i.e. $\tau_{s d}$ for pair $s-d$, is equal to the effective resistance between nodes $s$ and $d$ when the network is considered as an electrical network with link conductances equal to the link weights [4]. The total effective resistance of the network is also equal to the network criticality $\tau$. In this paper we use the terms network criticality and effective resistance interchangeably.

In order to simplify the structure of complex networks, one needs to have a good understanding of connectivity properties of a network as well as its robustness. In this paper our goal is to investigate $\hat{\tau}$ as a function of weight matrix $(W)$ and compare it with algebraic connectivity of a graph.

\section{NETWORK CRITICALITY AND ALGE- BRAIC CONNECTIVITY}




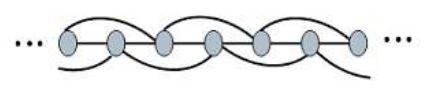

Extended Linear

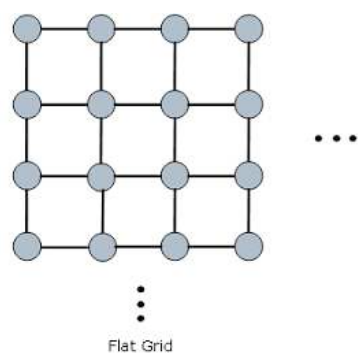

Figure 1: Extended Linear Graph (ELG) and Flat Grid Graph (FGG)

Fiedler [3] defined algebraic connectivity as the first nonzero eigenvalue $\left(\lambda_{2}\right)$ of the Laplacian matrix of a connected graph (recall that the first eigenvalue of Laplacian matrix for a connected graph is zero). Algebraic connectivity is a lower bound for node connectivity and link connectivity. Therefore, the further $\lambda_{2}$ is from zero, the higher the node and link connectivity of a graph.

In [11] we have shown that network criticality is bounded by the reciprocal of algebraic connectivity. More precisely:

$$
\frac{2}{(n-1) \lambda_{2}} \leq \hat{\tau} \leq \frac{2}{\lambda_{2}}
$$

This means that increasing the connectivity of a network, decreases the upper bound of network criticality, which potentially means more robustness.

\section{SIMULATION RESULTS}

In this section, motivated by [6], we provide an in-depth study of algebraic connectivity and network criticality (effective resistance). We used a diverse range of topologies, from structured graphs (linear graph, grid, Torus, etc) to random graphs and small-world networks. The quantities of interest for us are: network criticality, algebraic connectivity, average degree, and average node betweenness. In all of the experiments, we assume that all the link weights are equal. In fact, without loss of generality we assume $w_{i j}=1 \forall(i, j) \in E$.

\subsection{Deterministic Graphs}

While a complex network is usually not a regular or structured graph, frequently a large number of existing complex networks (for instance the network of proteins) can be built by joining some structured topologies. Therefore, in this section we study the behavior of the metrics of interest in deterministic and structured networks.

In the first experiment we consider extended linear graph (ELG), and flat grid graph (FGG) (see Fig. 1). In Fig. 2 we compare the behavior of network criticality, algebraic connectivity, average degree, and average node betweenness in ELG and FGG for different network sizes.

Fig. 2-a shows the behavior of network criticality for ELG and FGG. The criticality of ELG grows much faster than FGG. Fig. 2-b reveals that the algebraic connectivity of FGG is always better than ELG, that is the flat grid has better connectivity but the speed of decreasing the connectivity of the graph is much slower than increasing the network criticality. According to the Fig. 2-c, the average node degree of ELG approaches 4, and the average node degree

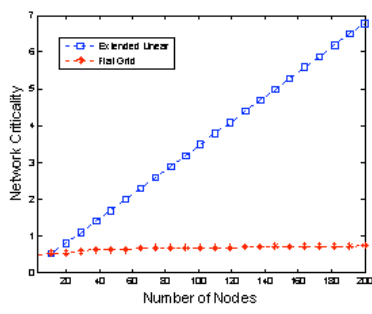

(a)

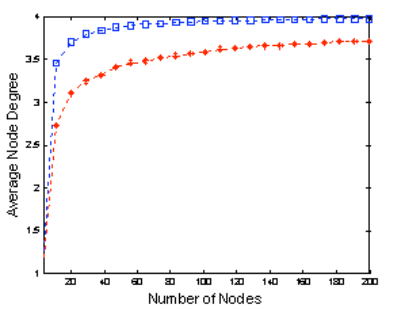

(c)

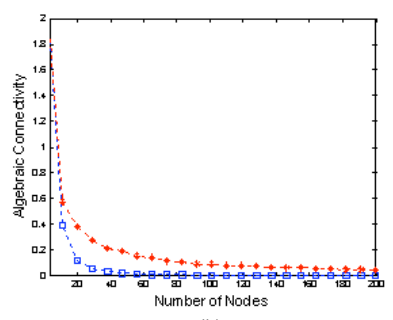

(b)

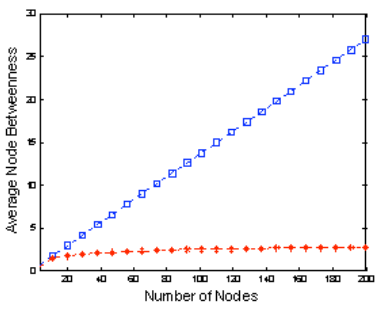

(d)
Figure 2: Comparison of Extended Linear and Flat Grid Topologies

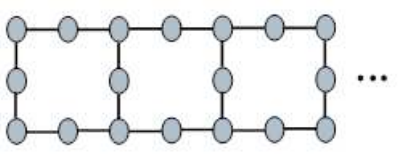

Ladder

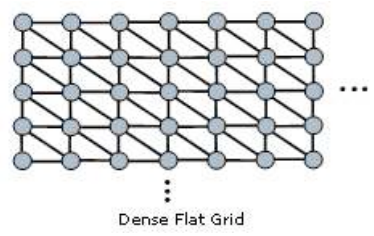

Dense Flat Grid
Figure 3: Topology of Ladder (LAG) and Dense Flat Grid Graph (DFG)

of FGG is between 3.5 and 4.0. Finally, Fig. 2-d shows that the average node betweenness of ELG is higher than that of FGG. The behavior of average node betweenness and network criticality in this experiment are very close, because the variations of node degree for both networks are small, therefore, according to equation 5 , the average node betweenness and network criticality are almost proportional.

This experiment reveals that while the changes in node degree and algebraic connectivity of ELG and FGG are relatively similar, there is a huge change in the behavior of network criticality, which means that network criticality captures some attributes of the graph that cannot be found in node degree and algebraic connectivity.

Fig. 3 shows the ladder and dense flat grid graph (DFG), which are two other topologies of interest. In Fig. 4, the behavior of FGG and DFG are compared. Fig. 4-a verifies that the network criticality of DFG is smaller than the network criticality of FGG with the same number of nodes. This is expected because the number of links in DFG is more than FGG. Therefore, if the objective of designing a network is to make it more robust in the sense that robustness is defined in this paper, we need to use DFG. According to Fig. 4-b the algebraic connectivity of FGG and DFG are very similar for all values of $n$. This means that if only the connectivity is critical in a network, then it is preferred to use FGG. In other words, if connectivity is the only concern, a DFG can be safely converted to a FGG.

Fig. 4-c shows the average node degree of FGG and DFG and Fig. 4-d shows the behavior of average node between- 


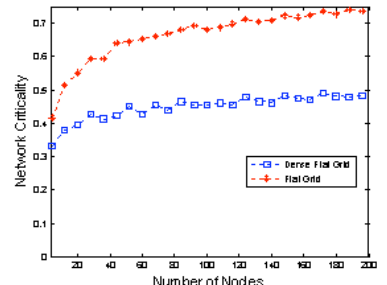

(a)

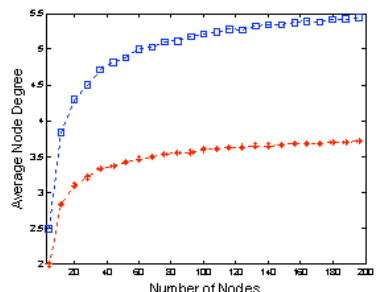

(c)

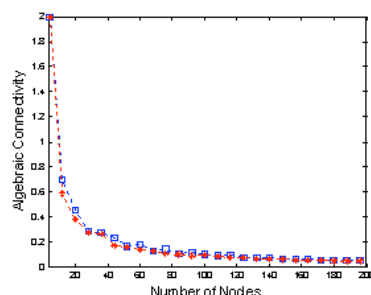

(b)

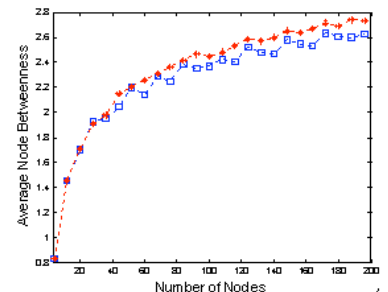

(d)
Figure 4: Comparison of Flat Grid and Dense Flat Grid Topologies

ness. According to this figure the average link betweenness for FGG and DFG are very similar. In other words, when the control of average node betweenness is the main objective, then it makes sense to use FGG with the same number of nodes. This will simplify the network significantly.

In the next experiment, we compare the behavior of extended linear graph (ELG) and ladder graph (LAG). According to Fig. 5, network criticality of LAG is slightly worse (larger) than the network criticality of ELG, while the algebraic connectivity of ELG and LAG behave similarly for different values of $n$. Average degree of ELG converges to to 4 , while the average node degree of LAG converges to a value less than 2.5. As the total number of links in a graph is approximately the number of nodes times the average node degree, we arrive at the result that when the networks grow in node size, the number of links in ELG is much more than LAG, in other words, a slight improvement in network criticality comes at the expense of a huge increase in the number of links (while the number of nodes in both networks are the same).

Now by looking at the result for average node betweenness, we find that the average node betweenness of ELG grows faster than LAG as $n$ grows. A final result is that in general by changing an ELG-like topology to a LAG, we do not lose too much, as a matter of fact the average node betweenness even decreases, while the network criticality (effective resistance) slightly increases. The average node betweenness has an important role in developing traffic engineering algorithms for communication networks [8].

Now we study the behavior of square torus graph (STG) and sparse flat graph (SFG) (Fig. 6). In Fig. 7 the behavior of STG and FGG are compared. Fig. 7-a shows that up to a certain number of nodes (around 16 nodes), the network criticality of STG decreases with increasing node number. It also reveals that for networks with less than 90 nodes STG has smaller network criticality than FGG with the same number of nodes. However, for larger networks FGG has better (smaller) network criticality. Fig. 7-b shows that algebraic connectivity of STG for networks with less than 80 nodes is better (larger) than that of FGG

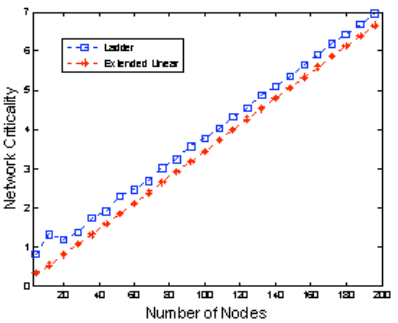

(a)

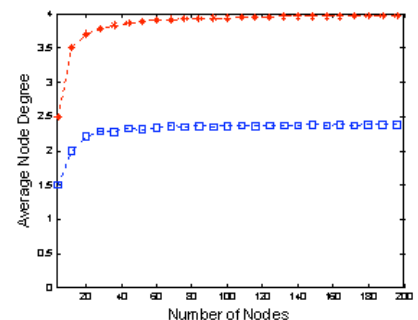

(c)

Figure 5: Comparison of Ladder and Extended Linear Graph Topologies
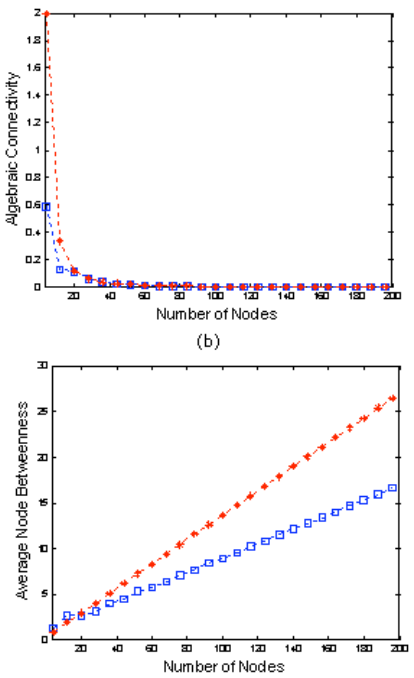

(d)

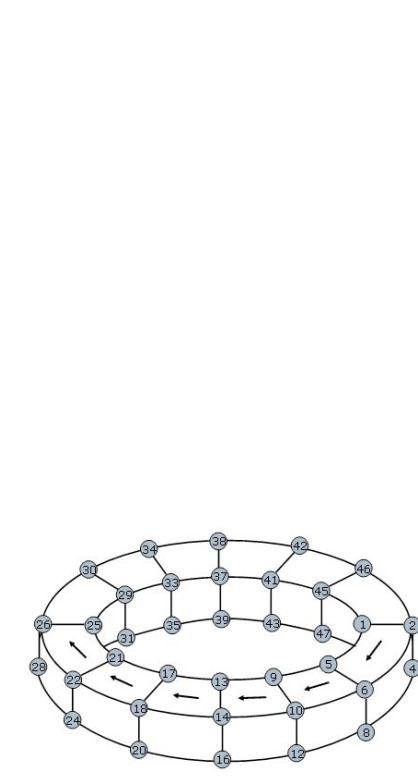

(a) STG

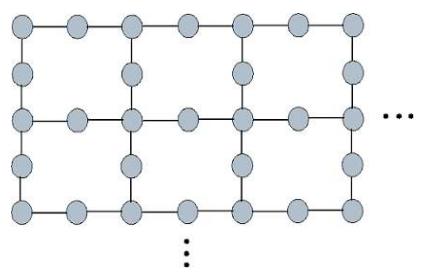

(b) SFG
Figure 6: Topology of Square Torus Graph (STG) and Sparse Flat Graph (SFG) 
(a)

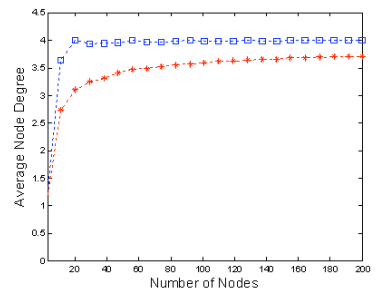

(c)

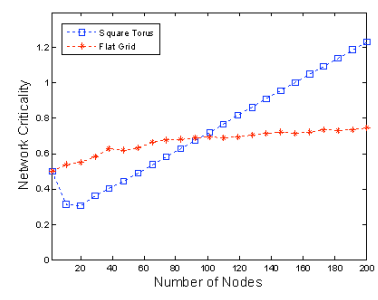

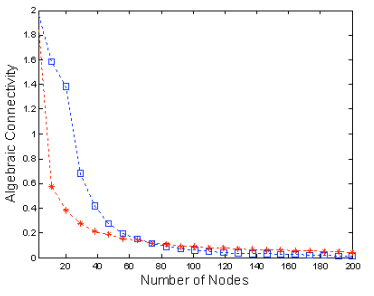

(b)

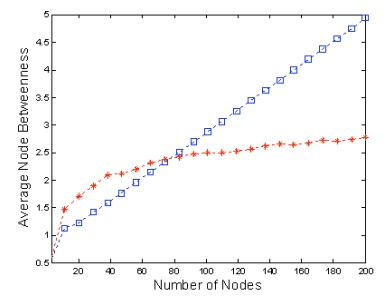

(d)
Figure 7: Behavior of STG and FGG

with the same number of nodes. It also shows that after 80 nodes algebraic connectivity of FGG is slightly better. However, the difference in the behavior of STG and FGG is more evident through their network criticality and average node betweenness. Fig. 7-c shows that average node degree of STG quickly approaches 4, while average node degree of FGG is always less that 4 and goes to 4 very slowly. According to the Fig. 7-d, this causes smaller average node betweenness for FGG networks when $80 \leq n \leq 90$, while the network criticality of STG is smaller for the networks with $80 \leq n \leq 90$ as shown in Fig. 7-a.

Finally, we study the robustness behavior of SFG and FGG. In Fig. 8 we compare the behavior of SFG with FGG. Fig. 8-c shows that for networks with large number of nodes, FGG has $45 \%$ more links than SFG. This increase in link density leads to a considerable improvement in network criticality, algebraic connectivity, and average node betweenness. Fig. 8-a shows that network criticality of FGG is much smaller than SFG. For $n=200$, the network criticality of SFG is 1.406 while it is 0.747 for FGG.

There are also some interesting points in the comparison of behavior of FGG, DFG and SFG using Fig. 8 and Fig. 4 . These figures show that moving from SFG to FGG generates more improvement in network metrics compared to moving from FGG to DFG, although in both movements we should increase number of links to the same extent.

\begin{tabular}{|c|c|c|c|}
\hline Quantity & SFG & FGG & DFG \\
\hline Average Degree & 2.55 & 3.71 & 5.43 \\
Network Criticality & 1.406 & 0.747 & 0.494 \\
\hline
\end{tabular}

Table 1: Numerical Comparison of Robustness in SFG, FGG, and DFG

Table 1 shows the average node degree and network criticality for these three topologies when $\mathrm{n}=200$. According to this table moving from SFG to FGG needs $45 \%$ increase

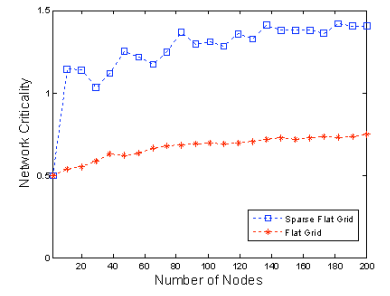

(a)

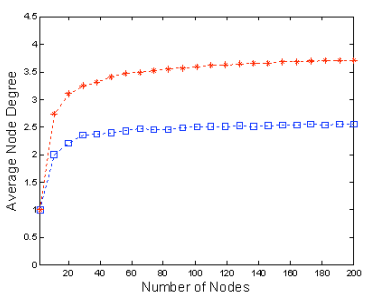

(c)

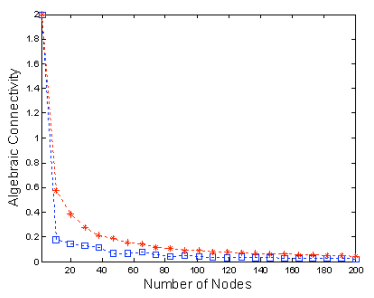

(b)

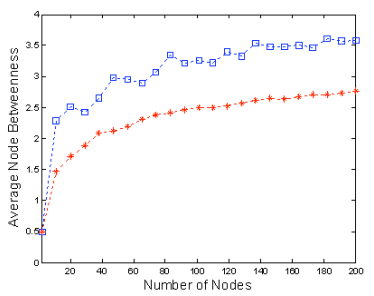

(d)
Figure 8: Behavior of SFG and FGG

in average degree and leads to $47 \%$ decrease in network criticality while moving from FGG to DFG needs an increase in average degree by $45 \%$, again but network criticality decreases only $34 \%$. In addition, comparison of Fig. 8-b with Fig. 4-b and Fig. 8-d with Fig. 4-d show that by moving form SFG to FGG we gain improvement in algebraic connectivity and average node betweenness, while there is almost no gain in these metrics by moving from FGG to DFG.

\subsection{Small-World Networks}

Small-world is a name for a subset of complex networks where despite the huge size of the network, the average path length between any two nodes is relatively small. The smallworld model proposed by Watts-Strogatz is the most studied small-world model [12]. Starting from a regular ring lattice, we connect each node with 2 s neighbors ( $s$ neighbors in each side), then we rewire each link with probability $p$. In rewiring process, we keep the first node of each link and reconnect the other end node (clockwise) with probability of $p$ to another node which is chosen randomly from the ring nodes in a way that sell-loops and parallel edges are not permitted. For $0.01 \leq p \leq 0.1$ small world characteristic appears (small average path length, large clustering coefficient). If we continue increasing the randomness by increasing $p$, the graph starts behaving more like a random graph. For $p=1$ we get a pure random graph (small average path length, small clustering coefficient).

In Fig. 9 variations of average network criticality and average algebraic connectivity with link connectivity for different values of $n$ are shown. Fig. 9-a and 9-b show the behavior of average network criticality and algebraic connectivity for $p=0.01$, and Figures 9-c and 9-d correspond to $p=0.1$. For each combination of $n$ and $p$, we have generated 10, 000 graphs. The value of $s$ is taken to be uniformly distributed between 1 to 10 units $(1 \leq s \leq 10)$. The results shown in Fig. 9 are the average values over all generated graphs.

One can see that average algebraic connectivity in both cases $(p=0.01$ and $p=0.1)$ and for different values of $n$ 


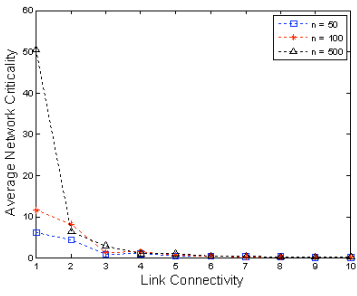

(a) $p=0.01$

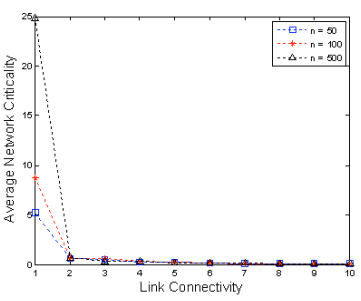

(c) $p=0.1$

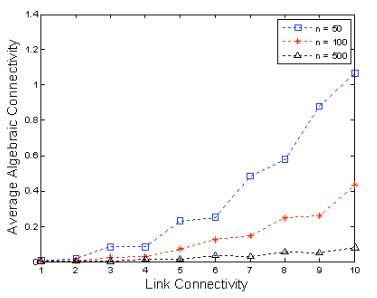

(b) $p=0.01$

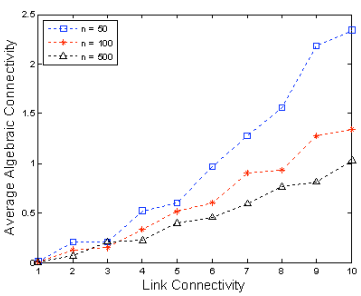

(d) $p=0.1$
Figure 9: Average Network Criticality and Algebraic Connectivity for Small-World Networks with $p=0.01$ and $p=0.1$

increases relatively smoothly with link connectivity. However, Fig. 9-a and 9-c show a significant change in average network criticality when link connectivity increases from 1 to 2. Therefore, if simplifying a small-world network leads to decreasing edge connectivity from 2 to 1 , this results in a huge jump (increase) in average network criticality (loosing robustness) which is not desirable. This fact is not captured through average algebraic connectivity charts.

Furthermore, when the link connectivity is more than 4, the value of average network criticality does not have a significant change even if the network grows from $n=50$ to $n=500$. In other words, for small-world networks, if the connectivity is fixed to a desirable value (this value depends on the probability $p$ ), increasing the network size does not cause dramatic change in network criticality.

\section{CONCLUSIONS}

In this paper we investigated the behavior of different graph theoretic measures on some well-known graph topologies. More specifically, we examined the effect of network size on network criticality, algebraic connectivity, average degree, and average node betweenness. We saw that there is no unique graph metric to satisfy both connectivity and robustness objectives while keeping a reasonable complexity. Each metric captures some attributes of the graph. It turns out that in order to design or simplify a network, we need to study the effect of all these graph metrics and choose the best network topology according to the requirements of the problem at hand.

\section{REFERENCES}

[1] A. H. Dekker and B. Colberet. The Symmetry Ratio of a Network. In Australasian symposium on Theory of computing, volume 41, pages 13-20, Newcastle, Australia, 2005. ACM International Conference Proceeding Series.
[2] A. H. Dekker and B. D. Colbert. Network Robustness and Graph Topology. Australasian Computer Science Conference, 26:359-368, Jan. 2004.

[3] M. Fiedler. Algebraic Connectivity of Graphs. Czechoslovak Math. Journal, 23(98):298-305, 1973.

[4] A. Ghosh, S. Boyd, and A. Saberi. Minimizing Effective Resistance of a Graph. SIAM Review, problems and techniques section, 50(1):37-66, February 2008.

[5] A. Jamakovic and P. V. Mieghem. On the Robustness of Complex Networks by Using the Algebraic Connectivity. In NETWORKING 2008 Ad Hoc and Sensor Networks, Wireless Networks, Next Generation Internet, pages 183-194. Springer Berlin / Heidelberg, May 2008.

[6] A. Jamakovic and S. Uhlig. On the Relationship between the Algebraic Connectivity and Graph Robustness to Node and Link Failures. In 3rd EuroNGI Conference, On Next Generation Internet Networks, pages 96-102, Trondheim, May 2007.

[7] M. Newman. A Measure of Betweenness Centrality Based on Random Walks. arXiv cond-mat/0309045., 2003.

[8] A. Tizghadam. Autonomic Core Network Management System. Phd thesis, University of Toronto, School of Electrical and Computer Engineering, April 2009.

[9] A. Tizghadam and A. Leon-Garcia. A Robust Routing Plan to Optimize Throughput in Core Networks. ITC20, Elsvier, pages 117-128, 2007.

[10] A. Tizghadam and A. Leon-Garcia. On Robust Traffic Engineering in Core Networks. In IEEE GLOBECOM, December 2008.

[11] A. Tizghadam and A. Leon-Garcia. Survival Value of Communication Networks. Infocom Workshop on Network Science for Communications (NetSciCom), April 2009.

[12] D. Watts and S. Strogatz. Collective Dynamics of Small-World Networks. Nature, (393):440-442, 1999. 